\title{
Three Extremal Problems for Hyperbolically Convex Functions
}

\author{
Roger W. Barnard, Kent Pearce, and G. Brock Williams
}

Dedicated to the memory of Walter Hengartner.

\begin{abstract}
Keywords. Hyperbolically convex functions, Julia variation.
\end{abstract}
2000 MSC. Primary 30C70.

\begin{abstract}
In this paper we apply a variational method to three extremal problems for hyperbolically convex functions posed by $\mathrm{Ma}$ and Minda and Pommerenke [7, 16]. We first consider the problem of extremizing $\operatorname{Re} \frac{f(z)}{z}$. We determine the minimal value and give a new proof of the maximal value previously determined by Ma and Minda. We also describe the geometry of the hyperbolically convex functions $f(z)=\alpha z+a_{2} z^{2}+a_{3} z^{3}+\ldots$ which maximize $\operatorname{Re} a_{3}$.
\end{abstract}

\section{Introduction}

A classical problem in Geometric Function Theory is to maximize the value of a given functional over a given class of analytic functions. Recent papers have extended this problem and its study to functionals on hyperbolically convex functions. In particular, these functions were studied by Beardon in [6], Ma and Minda in [7, 8], Solynin in [17, 18], Mejía and Pommerenke in $[9,10,11,12,13]$ and Mejía, Pommerenke, and Vasil'ev in [14]. This flurry of activity has produced a number of open problems and conjectures.

Recently, in $[2,4]$ we developed a variational technique for hyperbolically convex functions based on Julia's variational formula and applied it to several of these problems and conjectures.

For $z \in \mathbb{C}$, let $\operatorname{Re}\{z\}=$ real part $z$ and let $\mathbb{D}=\{z \in \mathbb{C}:|z|<1\}$. With the metric $\lambda(z)|d z|=\frac{2|d z|}{1-|z|^{2}}, \mathbb{D}$ forms the Poincaré model of the hyperbolic plane. In this model, hyperbolic geodesics in $\mathbb{D}$ are subarcs of Euclidean circles which intersect $\partial \mathbb{D}$ orthogonally. A set $S \subset \mathbb{D}$ is hyperbolically convex if for any two points $z_{1}$ and $z_{2}$ in $S$, the hyperbolic geodesic connecting $z_{1}$ to $z_{2}$ lies entirely inside of $S$. Important examples of hyperbolically convex regions are the fundamental domains of Fuchsian groups.

We will say that a function $f: \mathbb{D} \rightarrow \mathbb{D}$ is hyperbolically convex if $f$ is analytic and univalent on $\mathbb{D}$ and if $f(\mathbb{D})$ is hyperbolically convex. The set of all hyperbolically convex functions $f$ which satisfy $f(0)=0, f^{\prime}(0)>0$ will be denoted by $H$.

A hyperbolic polygon is a simply connected subset of $\mathbb{D}$, which contains the origin and which is bounded by a Jordan curve consisting of a finite collection of hyperbolic geodesics and arcs of the unit circle. The bounding geodesics will be referred to as proper sides. We will let $H^{\text {poly }}$ denote the subset of $H$ of all functions mapping $\mathbb{D}$ onto hyperbolic polygons. Further, we will let $H^{n}$ denote the subclass of $H^{\text {poly }}$ of all functions mapping $\mathbb{D}$ onto polygons with at most $n$

Version April 5, 2004.

$\underset{1}{\operatorname{ISSN}} 1617-9447 / \$ 2.50$ (C) 20XX Heldermann Verlag 
proper sides. It can be shown that $H^{\text {poly }}$ is dense in $H$. Moreover, $H \cup\{0\}$ and $H^{n} \cup\{0\}$, for each $n$, are compact.

Each function $f \in H$ satisfies Schwarz's Lemma and, hence, $f^{\prime}(0) \leq 1$. For $0<\alpha \leq 1$, let $H_{\alpha}=\left\{f \in H: f(z)=\alpha z+a_{2} z^{2}+a_{3} z^{3}+\cdots\right\}$. As an aside, we note that $H_{1}$ consists only of the identity map.

Ma and Minda [7, 8] and Mejía and Pommerenke [9] describe the geometry of the function

$$
k_{\alpha}(z) \equiv \frac{2 \alpha z}{(1-z)+\sqrt{(1-z)^{2}+4 \alpha^{2} z}}
$$

which belongs to $H_{\alpha}$ and which maps $\mathbb{D}$ to a hyperbolic polygon bounded by exactly one proper side. As a consequence of the normalizations, it can easily be seen that $H_{\alpha} \cap H^{1}$ consists solely of $k_{\alpha}$ and its rotations.

Ma and Minda [7] and Pommerenke [16] posed the following three problems for hyperbolically convex functions, whose solutions did not follow from the techniques in $[9,7,8,11,12,13]$.

Problem 1. Fix $0<\alpha<1$ and let $f \in H_{\alpha}$. For $z \in \mathbb{D} \backslash\{0\}$, find

$$
\min _{f \in H_{\alpha}} \operatorname{Re} \frac{f(z)}{z} \text {. }
$$

Problem 2. Fix $0<\alpha<1$ and let $f \in H_{\alpha}$ with $f(z)=\alpha z+a_{2} z^{2}+a_{3} z^{3}+\cdots$. Find

$$
\max _{f \in H_{\alpha}} \operatorname{Re} a_{3} \text {. }
$$

Problem 3. Let $f \in H$ with $f(z)=\alpha z+a_{2} z^{2}+a_{3} z^{3}+\cdots$. Find

$$
\max _{f \in H} \operatorname{Re} a_{3} \text {. }
$$

Applying the variational methods developed in $[2,4]$, we have the following resolutions for Problems 1, 2 and 3.

Theorem 1.1. For any $z \in \mathbb{D} \backslash\{0\}$ and fixed $0<\alpha<1$, let $f \in H_{\alpha}$ be extremal for $L(f)=\operatorname{Re} \frac{f(z)}{z}$ over $H_{\alpha}$. Then the extremal value (maximum or minimum) for $L$ over $H_{\alpha}$ can be obtained from a hyperbolically convex function $f$ which maps $\mathbb{D}$ onto a hyperbolic polygon with exactly one proper side. Specifically, for $z \in \mathbb{D} \backslash\{0\}$ and $0<\alpha<1$

$$
\max _{f \in H_{\alpha}} \operatorname{Re} \frac{f(z)}{z}=\frac{k_{\alpha}(r)}{r}, r=|z|
$$

and

$$
\min _{f \in H_{\alpha}} \operatorname{Re} \frac{f(z)}{z}=\frac{k_{\alpha}(-r)}{-r}, r=|z| .
$$

Theorem 1.2. Fix $0<\alpha<1$. Then the maximal value for $L(f)=\operatorname{Re} a_{3}$ over $H_{\alpha}$ is obtained by a hyperbolically convex function $f(z)=\alpha z+a_{2} z^{2}+a_{3} z^{3}+\cdots$ which maps $\mathbb{D}$ onto a hyperbolic polygon with at most two proper sides.

Theorem 1.3. The maximal value for $L(f)=\operatorname{Re} a_{3}$ over $H$ is obtained by a hyperbolically convex function $f(z)=\alpha z+a_{2} z^{2}+a_{3} z^{3}+\cdots$ which maps $\mathbb{D}$ onto a hyperbolic polygon with at most two proper sides.

Remark 1.1. The maximum value of $\operatorname{Re} \frac{f(z)}{z}$ for hyperbolically convex functions was first given using different methods by Ma and Minda [7]. Minda and Ma also observed in [7] that $k_{\alpha}$ cannot be extremal for (2) when $\alpha=1 / 2$. Hence, the reduction in Theorem 1.2 of the extremal function to a hyperbolically convex function with two proper sides is best possible. 


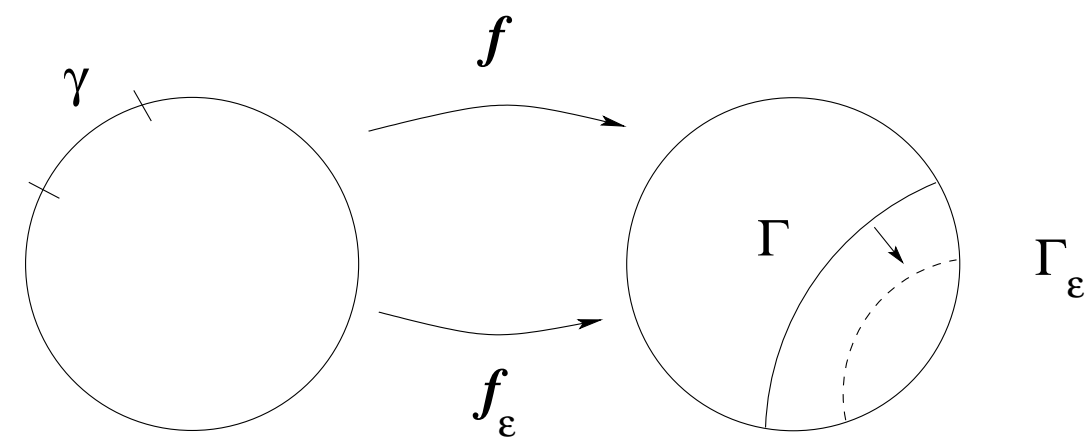

FIGURE 1. The variation produced by "pushing out" a side.

\section{Variations for $H^{\text {poly }}$}

As our primary tool for solving these three problems, we will modify an extension of the Julia variation as developed by the first author and J. Lewis [5, 3].

Let $\Omega$ be a region bounded by a piecewise analytic curve $\Gamma$ and $\phi(w)$ be a positive piecewise $C^{1}$ function on $\Gamma$, vanishing where $\Gamma$ is not analytic. Denote the outward pointing unit normal vector at each point $w$ where $\Gamma$ is smooth by $n(w)$. For $\epsilon$ near 0 , construct a new curve

$$
\Gamma_{\epsilon}=\{w+\epsilon \phi(w) n(w): w \in \Gamma\}
$$

and let $\Omega_{\epsilon}$ be the new region bounded by $\Gamma_{\epsilon}$.

Notice that if $\epsilon$ is sufficiently small and $\epsilon>0$, then $\Gamma$ is "pushed outside" the domain, while if $\epsilon<0$, then $\Gamma$ is "pushed inside" the domain. With the above notation, we state Julia's modification of the Hadamard variational formula; see [5] for details.

Lemma 2.1. Let $f$ be a conformal map from $\mathbb{D}$ onto $\Omega$ with $f(0)=0$, and suppose $f$ has a continuous extension to $\partial \mathbb{D}$, which we also denote by $f$. Then, a similarly normalized conformal map from $\mathbb{D}$ onto $\Omega_{\epsilon}$ is given by

$$
f_{\epsilon}(z)=f(z)+\frac{\epsilon z f^{\prime}(z)}{2 \pi} \int_{\partial \mathbb{D}} \frac{1+\xi z}{1-\xi z} d \Psi+o(\epsilon),
$$

where $d \Psi=\frac{\phi(f(\xi))}{\left|f^{\prime}(\xi)\right|} d \theta, \xi=e^{i \theta}$, and the $o(\epsilon)$ term is continuously differentiable in $\epsilon$ for each $z \in \mathbb{D}$. Furthermore, the change in mapping radius between $f_{\epsilon}$ and $f$ is given by

$$
\Delta m r\left(f_{\epsilon}, f\right)=\frac{\epsilon f^{\prime}(0)}{2 \pi} \int_{\partial \mathbb{D}} d \Psi+o(\epsilon) .
$$

Notice the restriction that $\phi$ vanish at the points of non-smoothness of $\partial \Omega$ is a strong one. It implies, for example, that while we can vary the sides of a hyperbolic polygon, we cannot move any of the vertices. However, it follows from the work of the first author and J. Lewis [3] that such an extended version of the Julia variation is possible (except at internal cusps, ie., where two sides meet at an angle of measure $2 \pi$, which do not occur for hyperbolically convex polygons). Moreover, they showed that the resulting function will agree with the Julia variational formula up to $o(\epsilon)$ terms.

In $[2,4]$, we proved the following two lemmas which describe variations for functions in $H^{\text {poly }}$ which preserve hyperbolic convexity. First, if $f$ maps onto a hyperbolically convex polygon $\Omega$ with a side $\Gamma$, we can "push" $\Gamma$ to a nearby geodesic $\Gamma_{\epsilon}$ in such a way that the varied function 


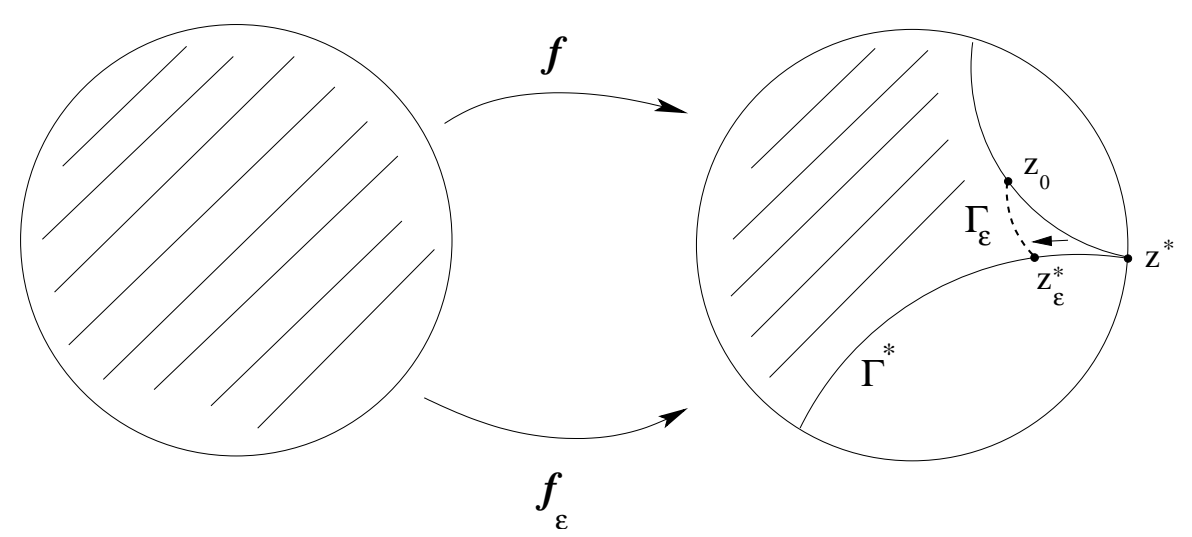

FiguRE 2. The variation produced by pushing in one end of a side.

$f_{\epsilon}$ will still be hyperbolically convex. Although the control $\phi$ can depend on $\epsilon$, it was shown in [2] that the variation in $\phi$ can be absorbed into the $o(\epsilon)$ terms. See Figure 1.

Lemma 2.2. Suppose $f \in H^{n}$ and $f$ is not constant. If $\Gamma=f(\gamma)$ is a proper side of $\Omega=f(\mathbb{D})$, then for $\epsilon$ sufficiently small there exists a variation $f_{\epsilon} \in H^{n}$ which "pushes" $\Gamma$ either in or out to a nearby geodesic $\Gamma_{\epsilon}$, where $\Gamma_{\epsilon} \rightarrow \Gamma$ as $\epsilon \rightarrow 0$. Moreover, $f_{\epsilon}$ agrees with the Julia Variational Formula up to o( $\epsilon)$ terms.

If $\Gamma$ intersects another side $\Gamma^{*}$ at $z^{*}$, and $z_{0} \in \Gamma$, then we can vary $f$ so as to replace the portion of $\Gamma$ between $z_{0}$ and $z^{*}$ with a new geodesic between $z_{0}$ and some $z_{\epsilon}^{*} \in \Gamma^{*}$. See Figure 2 .

Lemma 2.3. Suppose $f \in H^{n}, f$ is not constant, and $\Gamma=f(\gamma)$ is a proper side of $f(\mathbb{D})$ meeting a side $\Gamma^{*}$. Then, there exists a variation $f_{\epsilon} \in H_{n+1}$ which adds a side to $f(\mathbb{D})$ by pushing one end of $\Gamma$ to a nearby side $\Gamma_{\epsilon}$. That is, $f_{\epsilon}(\mathbb{D})$ is a hyperbolic polygon whose sides are the same as those of $f$, except that one end of $\Gamma$ has been replaced by $\Gamma_{\epsilon}$ and $\Gamma^{*}$ has been shortened. Moreover, $f_{\epsilon}$ agrees with the Julia Variational Formula up to o $(\epsilon)$ terms.

Remark 2.1. Notice that in order to maintain hyperbolic convexity, we can only push in the "end" of a side, that is, a subarc that ends at a vertex of the polygon. However, we can choose this subarc to be as long, or more importantly, as short, as we choose.

\section{Proofs}

3.1. Proof of Theorem 1.1. Choose a point $z \in \mathbb{D} \backslash\{0\}$. We will consider explicitly the problem of minimizing $\operatorname{Re}\left\{\frac{f(z)}{z}\right\}$ over $H_{\alpha}$. The case for maximizing $\operatorname{Re}\left\{\frac{f(z)}{z}\right\}$ over $H_{\alpha}$ is analogous.

Let $H_{\alpha}^{n}=H_{\alpha} \cap H^{n}$. Suppose that $f \in H_{\alpha}^{n}$ is extremal for (1) over $H_{\alpha}^{n}$ for some $n \geq 3$ and that $f(\mathbb{D})$ has at least three proper sides, say $\Gamma_{j}, j=1,2,3$. Let $\gamma_{j}=f^{-1}\left(\Gamma_{j}\right), j=1,2,3$. For each side $\Gamma_{j}$, apply the variation described in Lemma 2.2 to $\Gamma_{j}$, with control $\epsilon_{j}=\epsilon \lambda_{j}$. Let $f_{\epsilon}$ be the varied function obtained by varying each of the three sides $\Gamma_{j}, j=1,2,3$, of $f$. From Lemma 2.1, we have

$$
\frac{f_{\epsilon}(z)}{z}=\frac{f(z)}{z}+\epsilon \sum_{j=1}^{3} \frac{\lambda_{j}}{2 \pi} \int_{\gamma_{j}} f^{\prime}(z) \frac{1+\xi z}{1-\xi z} d \Psi+o(\epsilon)
$$


and

$$
\Delta m r\left(f_{\epsilon}, f\right)=\epsilon \sum_{j=1}^{3} \frac{\lambda_{j} \alpha}{2 \pi} \int_{\gamma_{j}} d \Psi+o(\epsilon)
$$

Hence, we can write

$$
L\left(f_{\epsilon}\right)=L(f)+\epsilon \operatorname{Re}\left\{\sum_{j=1}^{3} \frac{\lambda_{j}}{2 \pi} \int_{\gamma_{j}} f^{\prime}(z) \frac{1+\xi z}{1-\xi z} d \Psi+o(\epsilon)\right\} .
$$

If $\left.\frac{\partial \Delta m r\left(f_{\epsilon}, f\right)}{\Delta \epsilon}\right|_{\epsilon=0}=0$, then $f_{\epsilon}$ will also lie in $H_{\alpha}^{n}$ for $\epsilon$ sufficiently small. If in addition, $\left.\frac{\partial L\left(f_{\epsilon}\right)}{\partial \epsilon}\right|_{\epsilon=0}$ is non-zero, then the value of $L\left(f_{\epsilon}\right)$ can be made smaller than the value of $L(f)$ for some $\epsilon$ near 0 . Thus $f$ cannot be extremal for $(1)$ in $H_{\alpha}^{n}$. Using the above representations for $L\left(f_{\epsilon}\right)$ and $\Delta m r\left(f_{\epsilon}, f\right)$ we obtain

$$
\left.\frac{\partial L\left(f_{\epsilon}\right)}{\partial \epsilon}\right|_{\epsilon=0}=\operatorname{Re}\left\{\sum_{j=1}^{3} \frac{\lambda_{j}}{2 \pi} \int_{\gamma_{j}} f^{\prime}(z) \frac{1+\xi z}{1-\xi z} d \Psi\right\}
$$

and

$$
\left.\frac{\partial \Delta m r\left(f_{\epsilon}, f\right)}{\partial \epsilon}\right|_{\epsilon=0}=\sum_{j=1}^{3} \frac{\lambda_{j} \alpha}{2 \pi} \int_{\gamma_{j}} d \Psi
$$

Let $Q(\xi)=f^{\prime}(z) \frac{1+\xi z}{1-\xi z}$. As $d \Psi$ is real valued, we can apply the mean value theorem for integrals and rewrite $(6)$ as

$$
\left.\frac{\partial L\left(f_{\epsilon}\right)}{\partial \epsilon}\right|_{\epsilon=0}=\left\{\sum_{j=1}^{3} \frac{\lambda_{j}}{2 \pi} \operatorname{Re} Q\left(\xi_{j}\right) \int_{\gamma_{j}} d \Psi\right\}
$$

where $\xi_{j}$ belongs to the interior of the arc $\gamma_{j}$.

Since the kernel $Q$ of our integral is bilinear in $\xi$, it maps $\partial \mathbb{D}$ to a circle $\Lambda$. Hence, for $|z|<1$, not all three of the points $Q\left(\xi_{j}\right), j=1,2,3$, can have the same real part. Without loss of generality suppose that $\operatorname{Re} Q\left(\xi_{1}\right)>\operatorname{Re} Q\left(\xi_{2}\right)$. Then, we can push $\Gamma_{1}$ in, $\Gamma_{2}$ out (and not vary $\left.\Gamma_{3}\right)$ so as to decrease the value of $L\left(f_{\epsilon}\right)$ from the value of $L(f)$ within the class $H_{\alpha}^{n}$. Specifically, choose $\lambda_{1}<0<\lambda_{2}$ (and $\left.\lambda_{3}=0\right)$ so that $\left.\frac{\partial \Delta m r\left(f_{\epsilon}, f\right)}{\epsilon}\right|_{\epsilon=0}=0$ in (7). Then, we have from (8)

$$
\begin{aligned}
\left.\frac{\partial L\left(f_{\epsilon}\right)}{\partial \epsilon}\right|_{\epsilon=0} & =\operatorname{Re} Q\left(\xi_{1}\right) \frac{\lambda_{1}}{2 \pi} \int_{\gamma_{1}} d \Psi+\operatorname{Re} Q\left(\xi_{2}\right) \frac{\lambda_{2}}{2 \pi} \int_{\gamma_{2}} d \Psi \\
& <\operatorname{Re} Q\left(\xi_{1}\right)\left(\frac{\lambda_{1}}{2 \pi} \int_{\gamma_{1}} d \Psi+\frac{\lambda_{2}}{2 \pi} \int_{\gamma_{2}} d \Psi\right) \\
& =0 .
\end{aligned}
$$

Thus, $f$ is not extremal for $L$ in $H_{\alpha}^{n}$. Consequently, if $f$ is extremal in $H_{\alpha}^{n}, n \geq 3$, then $f \in H_{\alpha}^{2} \subset H_{\alpha}^{n}$, that is, the extremal $f$ can have at most two proper sides. 


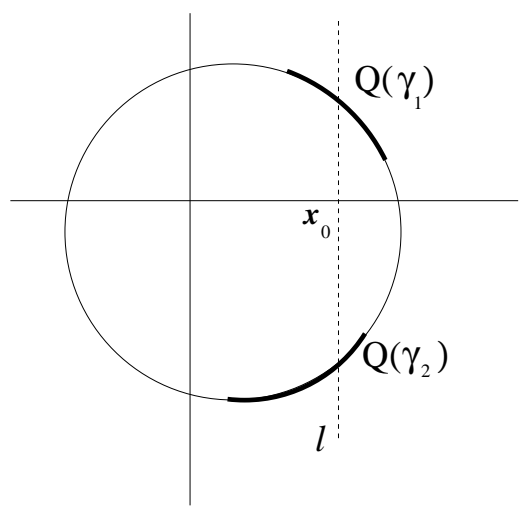

Figure 3. The endpoints of $Q\left(\gamma_{j}\right), j=1,2$, must lie on opposite sides of the line $l$ determined by $x=x_{0}=\operatorname{Re} Q\left(\xi_{1}\right)=\operatorname{Re} Q\left(\xi_{2}\right)$.

We will now argue that $f$ can actually have at most one side using an argument similar to the Step Down Lemma in [2]. Consider $H_{\alpha}^{n}, n \geq 3$, and let $f$ be extremal in $H_{\alpha}^{n}$ for (1). By the above argument, $f(\mathbb{D})$ can have at most two sides. Suppose $f(\mathbb{D})$ has exactly two proper sides, say $\Gamma_{j}, j=1,2$. As above, for each side $\Gamma_{j}$, apply the variation described in Lemma 2.2 to $\Gamma_{j}$, with control $\epsilon_{j}=\epsilon \lambda_{j}$. Let $f_{\epsilon}$ be the varied function obtained by varying each of the two sides $\Gamma_{j}, j=1,2$, of $f$. If in the formulation for $\left.\frac{\partial L\left(f_{\epsilon}\right)}{\partial \epsilon}\right|_{\epsilon=0}$ in (8), suitably modified to reflect only moving two sides, $\operatorname{Re} Q\left(\xi_{1}\right) \neq \operatorname{Re} Q\left(\xi_{2}\right)$, then the same argument used above eliminates $f$ from being extremal. So we conclude that $\operatorname{Re} Q\left(\xi_{1}\right)=\operatorname{Re} Q\left(\xi_{2}\right)=x_{0}$. Thus, for each proper side $\Gamma_{j}, j=1,2$, of $f(\mathbb{D})$, we must have that the image under the kernel $Q$ of the preimage of one endpoint of $\Gamma_{j}$ lies to the left of the vertical line $l$ determined by $x=x_{0}$ and the image under the kernel $Q$ of the preimage of other endpoint $\Gamma_{j}$ lies to the right of $l$. See Figure 3.

We consider the vertex $z_{0} \in \Gamma_{1}$ whose image under $Q \circ f^{-1}$ lies to the right of $l$. Apply the variation described in Lemma 2.3 at the vertex $z_{0}$ to add another side to $f(\mathbb{D})$, making sure the added side is short enough that its image under $Q \circ f^{-1}$ still lies completely to the right of $l$. At the same time, push the entire side $\Gamma_{2}$ out so that the mapping radius is preserved and $f_{\epsilon} \in H_{\alpha}^{2}$.

By the above variational argument, the newly varied function has a smaller value for $L$. But this means $f$ cannot be extremal. Thus, the extremal function for $L$ in $H_{\alpha}^{n}, n \geq 3$, cannot have two proper sides. It follows therefore that the extremal function in $H_{\alpha}^{n}$ can have at most one proper side.

Since $H_{\alpha}^{2} \subset H_{\alpha}^{n}$ for all $n \geq 3$, if $f$ is extremal in $H_{\alpha}^{n}$ and is an element of $H_{\alpha}^{2}$, it must be extremal in $H_{\alpha}^{2}$ as well. Thus, the extremal element in $H_{\alpha}^{2}$ has at most one proper side. As a result, the extremal value for $L$ in each $H_{\alpha}^{n}$ is achieved by the region with at most one proper side and hence the extremal value for $H_{\alpha}=\overline{\cup_{n \in \mathbb{N}} H_{\alpha}^{n}}$ is achieved by a region with at most one proper side.

Finally, it is clear that the range of $k_{\alpha}(z) / z$ is symmetric about the real axis. It can be shown for fixed $r, 0<r<1$, that $\operatorname{Re}\left\{k_{\alpha}\left(r e^{i \theta}\right) / r e^{i \theta}\right\}$ is a monotonically decreasing function of $\theta, 0<\theta<\pi$. Hence, the minimum value of $L$ over $H_{\alpha}$ is achieved at $-k_{\alpha}(-r) / r, r=|z|$. A similar argument shows that the maximum occurs at $k_{\alpha}(r) / r$.

3.2. Proof of Theorem 1.2. Next we fix $0<\alpha \leq 1$ and employ a similar argument to show that the maximum value of

$$
L(f)=\operatorname{Re} a_{3}
$$



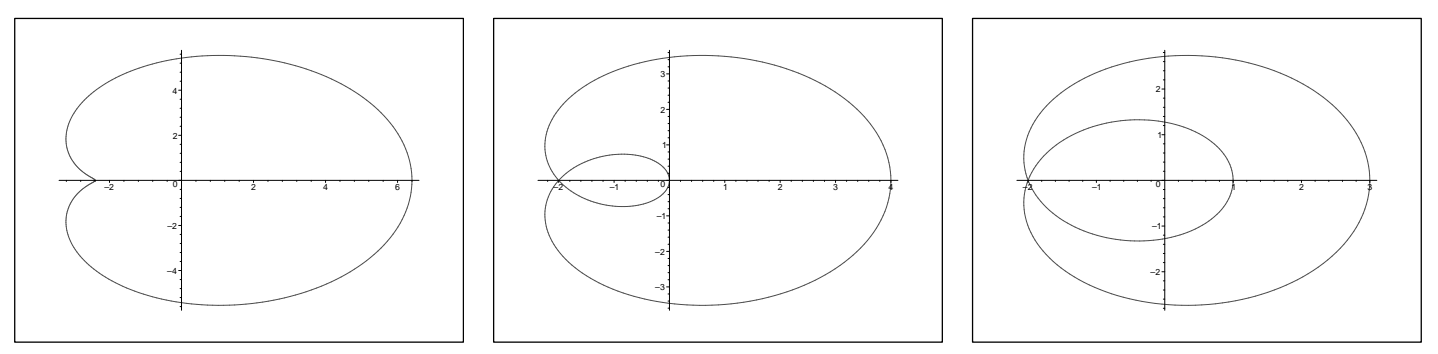

Figure 4. The cardioids resulting from $a_{2}=1.1$ (left), 0.5 (center), and 0.25 (right).

for a function $f(z)=\alpha z+a_{2} z^{2}+a_{3} z^{3}+\ldots$ in $H_{\alpha}$ is obtained by a function mapping onto a domain with at most two sides. Suppose first that $f \in H_{n}^{\alpha}$ is extremal over $H_{n}^{\alpha}$ for some $n \geq 5$ and $f(\mathbb{D})$ has at least five proper sides $\Gamma_{j}, j=1, \ldots, 5$. Let $\gamma_{j}$ be the preimage in $\partial \mathbb{D}$ of $\Gamma_{j}$, $j=1, \ldots, 5$.

If we apply the variation of Lemma 2.2 to each $\Gamma_{j}$ with control $\epsilon_{j}=\epsilon \lambda_{j}$, then we produce a hyperbolically convex function defined by

$$
f_{\epsilon}(z)=f(z)+\epsilon z f^{\prime}(z) \sum_{j=1}^{5} \frac{\lambda_{j}}{2 \pi} \int_{\gamma_{j}} \frac{1+\xi z}{1-\xi z} d \Psi+o(\epsilon) .
$$

Expanding $\frac{1+\xi z}{1-\xi z}$ as a series, we see

$$
f_{\epsilon}(z)=f(z)+\epsilon z f^{\prime}(z) \sum_{j=1}^{5} \frac{\lambda_{j}}{2 \pi} \int_{\gamma_{j}}\left(1+2 \xi z+2 \xi^{2} z^{2}+2 \xi^{3} z^{2}+\ldots\right) d \Psi+o(\epsilon) .
$$

Now since $z f^{\prime}(z)=\alpha z+2 a_{2} z^{2}+3 a_{3} z^{3}+\ldots$, we have

$$
f_{\epsilon}(z)=f(z)+\epsilon \sum_{j=1}^{5} \frac{\lambda_{j}}{2 \pi} \int_{\gamma_{j}}\left(\alpha z+2\left(a_{2}+\alpha \xi\right) z^{2}+\left(3 a_{3}+4 a_{2} \xi+2 \alpha \xi^{2}\right) z^{3}+\ldots\right) d \Psi+o(\epsilon) .
$$

Finally, gathering the powers of $z$, we arrive at

$$
\begin{aligned}
f_{\epsilon}(z)=\alpha & \left(1+\epsilon \sum_{j=1}^{5} \frac{\lambda_{j}}{2 \pi} \int_{\gamma_{j}} d \Psi\right) z+\left(a_{2}+\epsilon \sum_{j=1}^{5} \frac{\lambda_{j}}{2 \pi} \int_{\gamma_{j}} 2\left(a_{2}+\alpha \xi\right) d \Psi\right) z^{2} \\
& +\left(a_{3}+\epsilon \sum_{j=1}^{5} \frac{\lambda_{j}}{2 \pi} \int_{\gamma_{j}}\left(3 a_{3}+4 a_{2} \xi+2 \alpha \xi^{2}\right) d \Psi\right) z^{3}+\cdots+o(\epsilon) .
\end{aligned}
$$

Consequently,

$$
\left.\frac{\partial}{\partial \epsilon} \operatorname{Re} L\left(f_{\epsilon}\right)\right|_{\epsilon=0}=\operatorname{Re} \sum_{j=1}^{5} \frac{\lambda_{j}}{2 \pi} \int_{\gamma_{j}}\left(3 a_{3}+4 a_{2} \xi+2 \alpha \xi^{2}\right) d \Psi
$$

and

$$
\left.\frac{\partial \Delta m r\left(f_{\epsilon}, f\right)}{\partial \epsilon}\right|_{\epsilon=0}=\sum_{j=1}^{5} \frac{\alpha \lambda_{j}}{2 \pi} \int_{\gamma_{j}} d \Psi .
$$

Now notice that the kernel $Q(\xi)=3 a_{3}+4 a_{2} \xi+2 \alpha \xi^{2}$ of the integral in (11) maps the unit circle $\partial \mathbb{D}$ onto a cardioid. See Figure 4 . The shape of the cardioid depends on $a_{2}$ and $\alpha$, but a 


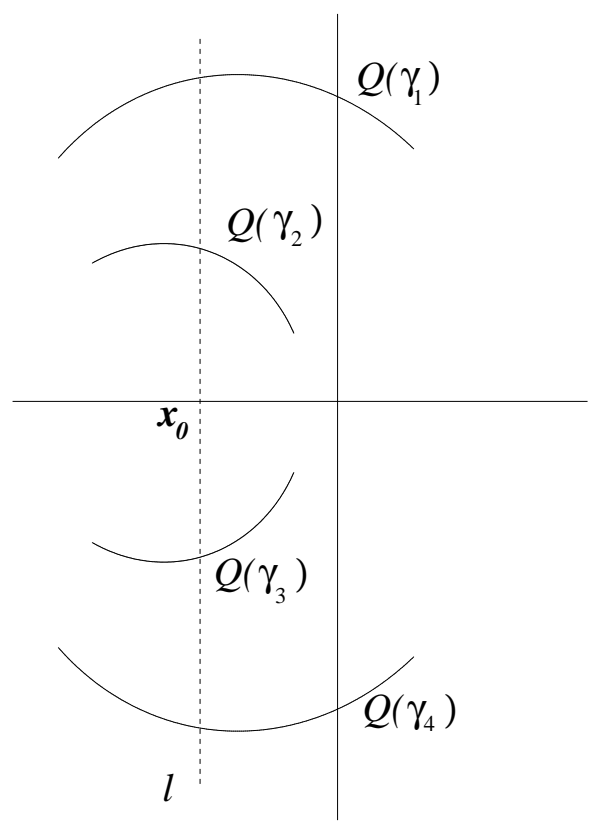

FiguRE 5. If the extremal function has four sides, then each curve $Q\left(\gamma_{j}\right)$ must cross the vertical line $l$ given by $x=x_{0}=\operatorname{Re} Q\left(\xi_{j}\right), j=1, \ldots, 4$.

simple argument shows that no vertical line intersects the cardioid more than four times. Thus if we apply the Mean Value Theorem to each of the integrals $\int_{\gamma_{j}}\left(3 a_{3}+4 a_{2} \xi+2 \alpha \xi^{2}\right) d \Psi$, we have

$$
\operatorname{Re} \frac{\lambda_{j}}{2 \pi} \int_{\gamma_{j}}\left(3 a_{3}+4 a_{2} \xi+2 \alpha \xi^{2}\right) d \Psi=\operatorname{Re} \frac{\lambda_{j} Q\left(\xi_{j}\right)}{2 \pi} \int_{\gamma_{j}} d \Psi
$$

for some $\xi_{j} \in \gamma_{j}, j=1, \ldots, 5$.

Suppose the values of $\operatorname{Re} Q\left(\xi_{j}\right)$ are not the same for all $j$, say $\operatorname{Re} Q\left(\xi_{1}\right)<\operatorname{Re} Q\left(\xi_{2}\right)$. Then just as in the proof of Theorem 1.1, we can push $\Gamma_{1}$ in and $\Gamma_{2}$ out, preserving the mapping radius to produce a new map $f_{\epsilon} \in H_{n}^{\alpha}$ with

$$
\left.\frac{\partial}{\partial \epsilon} L\left(f_{\epsilon}\right)\right|_{\epsilon=0}>0
$$

Consequently, since $f$ is extremal, then $\operatorname{Re} Q\left(\xi_{1}\right)=\cdots=\operatorname{Re} Q\left(\xi_{5}\right)$. But that means the vertical line $l$ determined by $x=x_{0}=\operatorname{Re} Q\left(\xi_{1}\right)=\cdots=\operatorname{Re} Q\left(\xi_{5}\right)$ intersects the cardioid $Q(\partial \mathbb{D})$ in five distinct points. This contradiction implies $f$ can have at most four sides.

Now notice that if $f$ is an extremal function with exactly four sides, then as above, $\operatorname{Re} Q\left(\xi_{j}\right)=$ $x_{0}$ for each $j=1, \ldots, 4$, as otherwise we could vary two sides and increase the value of $L(f)$. Geometrically, this means that each curve $Q\left(\gamma_{j}\right)$ must cross the vertical line $l$. See Figure 5.

As a result, one endpoint of each curve $Q\left(\gamma_{j}\right)$ must lie to the left of $l$, and we can use the variation described in Lemma 2.3 to add a new side $\Gamma_{\epsilon}$ at the end of one of the current sides. If $\Gamma_{\epsilon}$ is sufficiently short and $\gamma_{\epsilon}=f^{-1}\left(\Gamma_{\epsilon}\right)$, then $Q\left(\gamma_{\epsilon}\right)$ will lie completely to the left of $l$. Thus there exists $\xi_{\epsilon}$ so that

$$
\operatorname{Re} \int_{\gamma_{\epsilon}} Q(\xi) d \Psi=\operatorname{Re} Q\left(\xi_{\epsilon}\right) \int_{\gamma_{\epsilon}} d \Psi
$$




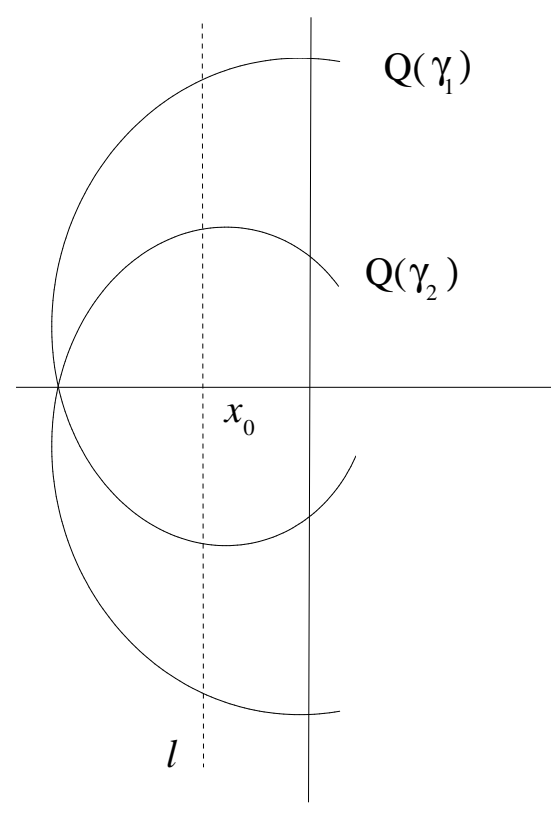

Figure 6. For a function with two sides, all four endpoints of $Q\left(\gamma_{j}\right)$ may lie on the same side of the line $l$ determined by $x=x_{0}=\operatorname{Re} Q\left(\gamma_{1}\right)=\operatorname{Re} Q\left(\gamma_{2}\right)$.

and

$$
\operatorname{Re} Q\left(\xi_{\epsilon}\right)<x_{0}
$$

Then arguing as above, we produce a new function $f_{\epsilon} \in H_{\alpha}^{5}$ with five sides and $L\left(f_{\epsilon}\right)>L(f)$.

But the extremal function in $H_{\alpha}^{5}$ has at most four sides. This contradiction means the extremal function in $H_{\alpha}^{n}, n \geq 3$ can have at most three sides.

However, notice that if the extremal function has three sides, then we must still have one endpoint of $Q\left(\gamma_{j}\right)$ on the left of the line $l$ for some $j=1,2,3$. Thus the argument above can be repeated to show that $f$ can have at most two sides.

On the other hand, if $f$ has two sides, it is certainly possible for all four endpoints of $Q\left(\gamma_{j}\right)$, $j=1,2$ to lie to the right of $l$, precluding the reduction to only one side. See Figure 6 . This is entirely to be expected, of course, as Ma and Minda have shown the extremal domain for $\alpha=1 / 2$ has more than one side [7].

Since the extremal function in $H_{\alpha}^{n}$ for each $n \geq 2$ has at most two sides and $\cup_{n} H_{\alpha}^{n}$ is dense in $H_{\alpha}$, the maximum value of $L(f)$ is obtained by function mapping onto a domain with at most two proper sides.

3.3. Proof of Theorem 1.3. In Theorem 1.3 we consider the same functional as in Theorem 1.2 , but we maximize not only over all $f \in H_{\alpha}$, but also over all $\alpha$. The arguments used in the proof above still apply, only we no longer need to ensure

$$
\left.\frac{\partial \Delta m r\left(f_{\epsilon}, f\right)}{\partial \epsilon}\right|_{\epsilon=0}=\sum_{j=1}^{5} \frac{\lambda_{j} \alpha}{2 \pi} \int_{\gamma_{j}} d \Psi=0
$$

when we perform a variation. In any case, we see there is an extremal function mapping onto a region with at most two sides. 


\section{References}

[1] Roger W. Barnard and John L. Lewis. Subordination theorems for some classes of starlike functions. Pacific J. Math., 56(2):333-366, 1975.

[2] Roger W. Barnard, Leah B. Cole, Kent Pearce, and G. Brock Williams. Sharp bounds for the Schwarzian derivative for hyperbolically convex functions. Preprint, 2003.

[3] Roger Barnard and John L. Lewis, Subordination theorems for some classes of starlike functions, Pacific J. Math. 56 (1975), no. 2, 333-366. MR52 \#721

[4] Roger W. Barnard, G. Lenny Ornas, and Kent Pearce. A variational method for hyperbolically convex functions. Preprint, 2003.

[5] Roger W. Barnard and Glenn Schober, Möbius transformations of convex mappings, Complex Variables Theory Appl. 3 (1984), no. 1-3, 55-69. MR85j:30012

[6] A. Beardon, Geometry of discrete groups, Springer-Verlag, Berlin, 1983.

[7] William Ma and David Minda, Hyperbolically convex functions, Ann. Polon. Math. 60 (1994), no. 1, 81-100. MR95k:30037

[8] William Ma and David Minda, Hyperbolically convex functions. II, Ann. Polon. Math. 71 (1999), no. 3, 273-285. MR2000j:30020

[9] Diego Mejía and Christian Pommerenke, On hyperbolically convex functions, J. Geom. Anal. 10 (2000), no. $2,365-378$.

[10] Diego Mejía and Christian Pommerenke, On spherically convex univalent functions, Michigan Math. J. 47 (2000), no. 1, 163-172. MR2001a:30013

[11] Diego Mejía and Christian Pommerenke, Sobre la derivada Schawarziana de aplicaciones conformes hiperbólicamente, Revista Colombiana de Matemáticas 35 (2001), no. 2, 51-60.

[12] Diego Mejía and Christian Pommerenke, Hyperbolically convex functions, dimension and capacity, Complex Var. Theory Appl. 47 (2002), no. 9, 803-814. MR2003f:30017

[13] Diego Mejía and Christian Pommerenke, On the derivative of hyperbolically convex functions, Ann. Acad. Sci. Fenn. Math. 27 (2002), no. 1, 47-56.

[14] Diego Mejía, Christian Pommerenke, and Alexander Vasil'ev, Distortion theorems for hyperbolically convex functions, Complex Variables Theory Appl. 44 (2001), no. 2, 117-130. MR2003e:30025

[15] Michalska, Malgorzata, Dmitri V. Prokhorov, and Jan Szynal, The compositions of hyperbolic triangle mappings, Complex Variables Theory Appl. 43 (2000), no. 2, 179-186. MR 2001m:30008

[16] Christian Pommerenke, private communication.

[17] A. Yu. Solynin, Some extremal problems on the hyperbolic polygons, Complex Variables Theory Appl. 36 (1998), no. 3, 207-231. MR99j:30030

[18] A. Yu. Solynin, Moduli and extremal metric problems, Algebra i Analiz 11 (1999), no. 1, 3-86, translation in St. Petersburg Math. J. 11 (2000), no. 1, 1-65. MR2001b:30058

Roger W. Barnard AdDREss: Department of Mathematics and Statistics, Texas Tech University, Lubbock, $T X 79409$

E-MAIL: barnard@math.ttu.edu

Kent Pearce Address: Department of Mathematics and Statistics, Texas Tech University, Lubbock, TX 79409

E-MAIL: pearce@math.ttu.edu

G. Brock Williams AdDress: Department of Mathematics and Statistics, Texas Tech University, Lubbock, TX 79409

E-MAIL: williams@math.ttu.edu 